\title{
Prognostic significance of microvessel density and other variables in Japanese and British patients with primary invasive breast cancer
}

\author{
T Kato*, I,2, G Steers' ${ }^{2}$, L Campo ${ }^{2}$, H Roberts², RD Leek², H Turley², T Kimura', S Kameoka', T Nishikawa ${ }^{3}$, \\ M Kobayashi" ${ }^{4}$ AL Harris ${ }^{5}, K_{C}$ Gatter $^{2}$ and F Pezzella ${ }^{2}$
}

'Department of Surgery II, School of Medicine, Tokyo Women's Medical University, 8-I Kawadacho, Shinjuku-ku, Tokyo I62-8666, Japan; '2Cancer Research UK Tumor Pathology Group, Nuffield Department of Clinical Laboratory Sciences, University of Oxford, John Radcliffe Hospital, Headington, Oxford OX3 9DU, UK; ${ }^{3}$ Department of Surgical Pathology, School of Medicine, Tokyo Women's Medical University, 8-I Kawadacho, Shinjuku-ku, Tokyo 162-8666, Japan; ${ }^{4}$ Department of Pathology, School of Medicine, Tokyo Women 's Medical University, 8-I Kawadacho, Shinjuku-ku, Tokyo I 62-8666, Japan; ${ }^{5}$ Cancer Research UK Molecular Oncology Laboratory, Institute of Molecular Medicine, John Radcliffe Hospital, Headington, Oxford OX3 9DU, UK

\begin{abstract}
The purpose of this study is to investigate the associations of microvessel density (MVD) and other pathological variables with survival, and whether they accounted for survival differences between Japanese and British patients. One hundred seventy-three Japanese and 184 British patients were included in the study. British patients were significantly older $(56.3 \pm$ II .4 years vs $52.5 \pm$ I 2.9 years; $P<0.01)$ and had smaller tumours $(2.2 \pm 1.3$ vs $2.7 \pm 1.8 \mathrm{~cm} ; P<0.01)$, which were more frequently oestrogen receptor positive (78.8 vs $57.2 \%, P<0.0$ I), had more grade III tumours $(29.9$ vs $21.4 \%, P=0.04)$ and more infiltrating lobular carcinomas $($ I 3.6 vs $4.0 \%, P<0.01)$ and a higher MVD compared with Japanese patients $(57.9 \pm 19.8$ vs $53.2 \pm 18.6 ; P=0.01)$. However, no difference in the prevalence of lymph-node metastasis was found between them (39.I vs 37.5\%, $P=0.75$ ). Younger British patients (age $<50$ years) had the highest MVD compared with Japanese and older British patients $(P<0.01)$. Japanese patients were proportionately more likely to receive chemotherapy than endocrine therapy $(P<0.01)$. British patients had a significantly worse relapse-free survival and overall survival compared with Japanese patients, after statistical adjustment for variables (hazard ratio $=2.1,2.4, P<0.01$, $P<0.0$ I, respectively), especially, in T2 stage, low MVD and older subgroup (HR: 3.6, 5.0; 3.I, 3.3; 3.2, 3.9, respectively), but only in ER negative cases $(P=0.04, P=0.01$, respectively). The present study shows that MVD contributes to the Japanese-British disparity in breast cancer. However, the MVD variability did not explain the survival differences between Japanese and British patients. British Journal of Cancer (2007) 97, I277-1286. doi:I0.1038/sj.bjc.66040 I5 www.bjcancer.com

Published online 9 October 2007

(c) 2007 Cancer Research UK
\end{abstract}

Keywords: angiogenesis; breast cancer; microvessel density; international differences

There have been many reports about racial disparities in breast cancer incidence and outcomes. Asian-American and Japanese patients tend to have a lower incidence of breast cancer and have a better prognosis than Caucasians (Yonemoto, 1980; Sakamoto et al, 1981; Natarajan et al, 1988; Tominaga and Kuroishi, 1995; Boyer-Chammard et al, 1999; Braun et al, 2004). Age-adjusted incidence rate for breast cancer among Japanese patients has markedly increased 1.9-fold from 1978 to 1998 (age-adjusted incidence rate: 17.9, 33.8, respectively) (The Research Group for Population-based Cancer Registration in Japan, 1999, 2003; Tamakoshi et al, 2005) and age-adjusted death rate has increased moderately (Tominaga and Kuroishi, 1995), but is still much lower compared with those of Caucasians (Tominaga and Kuroishi, 1995; Kawamura and Sobue, 2005). Racial differences in age-adjusted mortality rates are likely to be due to many factors, such as genetics, diet, reproductive patterns,

*Correspondence: Dr T Kato, Onuma Hospital, 2-10-2, Higashimizumoto, Katsushika-ku, Tokyo I25-0033, Japan.

E-mail: t-kato@bd5.so-net.ne.jp

Received 12 April 2007; revised 5 September 2007; accepted 7 September 2007; published online 9 October 2007 socioeconomic status, geographic and environmental exposures, and other unidentified cultural or biological factors (Iscovich et al, 1989; Claus et al, 1990; Chaudary et al, 1991; Gordon et al, 1992; Simon and Severson, 1996; Tamakoshi et al, 2005). In spite of numerous studies, the reason for such disparities has not been identified.

In general, angiogenesis facilitates both tumour growth and progression and it contributes to the aggressiveness of tumours. Some studies have suggested that microvessel density (MVD) as a measure of tumour angiogenesis is an independent and highly significant prognostic factor, for both node-negative and -positive patients (Weidner et al, 1991; Fox et al, 1995; Kato et al, 1999; Tsutsui et al, 2003). However, the numbers of microvessels counted by many investigators differs between studies, which also varied in patient selection, the antibody used to detect endothelial cells, sample size, method of counting microvessels, and race. Since the number of microvessels reported by Western investigators appears to be higher than that by Japanese (Axelsson et al, 1995; Costello et al, 1995; Ogawa et al, 1995; Kato et al, 1999) and green tea, consumed many times daily by the average Japanese, inhibits vascular endothelial growth factor induction in human breast cancer cells (Sartippour et al, 2002), we hypothesised that 
differences in MVD might contribute to the Japanese and British disparities in breast cancer outcomes.

This study was undertaken to investigate the associations of MVD and other variables with survival, and whether they are associated with survival differences between Japanese and British patients.

\section{PATIENTS AND METHODS}

Two hundred and seventeen Japanese and 219 British patients were studied, excluding patients with non-invasive, Stage IV, bilateral, male, or inflammatory cancers, to investigate the characteristics of primary operable invasive female breast cancer. They had undergone breast cancer surgery at the Tokyo Women's Medical University Hospital or the John Radcliffe Hospital, Oxford between 1991 and 1993. These hospitals are tertiary referral centres. However, cases where insufficient material remained in the tissue blocks for immunohistochemical evaluation of factor VIII-related antigen were excluded. Eleven Japanese and 14 British samples for study were identified retrospectively as having no carcinoma and paraffin-embedded tissue blocks of 33 Japanese and 21 British cases were insufficient because they have been used for other research. This left 173 cases in the Japanese group and 184 cases in British group for pathological analyses.

Follow-up was from medical records. Cause of death was analysed for breast cancer alone and cases who died from other disease was defined as censored. The date of the last note in the medical record in Tokyo was 2000, while in Oxford it was 2001. Oestrogen receptor (ER) content were determined biochemically using the dextran-coated charcoal method in Tokyo and Oxford. Tumours were classified as ER-positive if the content exceeded $5 \mathrm{fmol} \mu \mathrm{g}^{-1}$ protein. Patients' ages were categorised as age $<50$ years (younger group) and age $\geqq 50$ years (older group).

\section{Pathological studies}

The pathologic specimens from both hospitals were reviewed by the same investigator (TK) without any knowledge of the eventual clinical outcome. Conventional pathological features were observed and recorded, including lymph-node status. Operative/ pathological size was used for T stage in Japanese and pathological size for British patients. Grade was determined by the method of Elston and Ellis grade (Elston and Ellis, 1991). Vascular invasion was assessed on haematoxylin and eosin (H\&E) sections.

\section{Immunocytochemical techniques}

Serial sections were prepared from representative formalin-fixed and paraffin-embedded tissue blocks from this series of breast cancers. Japanese sections were fixed in $20 \%$ formalin for $48 \mathrm{~h}$, while British ones in $10 \%$ formalin for the same time. The other processing was similar in Tokyo and Oxford. Tissue samples of $5 \mu \mathrm{m}$ thick sections stained with $\mathrm{H} \& \mathrm{E}$ were assessed histopathologically and were used to select the maximal area of the invasive components. Immunostains for factor VIII-related antigen were performed on paraffin sections using the streptavidin-biotinimmunoperoxidase method as described previously (Kato et al, 1999, 2003). Briefly, formalin-fixed, paraffin-embedded sections were de-waxed in $100 \%$ Citroclear, rehydrated through graded $100 \%$ industrial methylated spirit series, and immunostaining was performed using a polyclonal antibody (von Willebrand factor, Dako, Copenhagen, Denmark) applied at $1: 200$ for $1 \mathrm{~h}$ at room temperature. Analysis of all slides of both Japanese and British cases for $\mathrm{H} \& \mathrm{E}$ and factor VIII-related antigen staining was performed by the same method and at same institute (John Radcliffe Hospital, Headington, Oxford, UK) and both clinical and pathological studies were done in Tokyo.
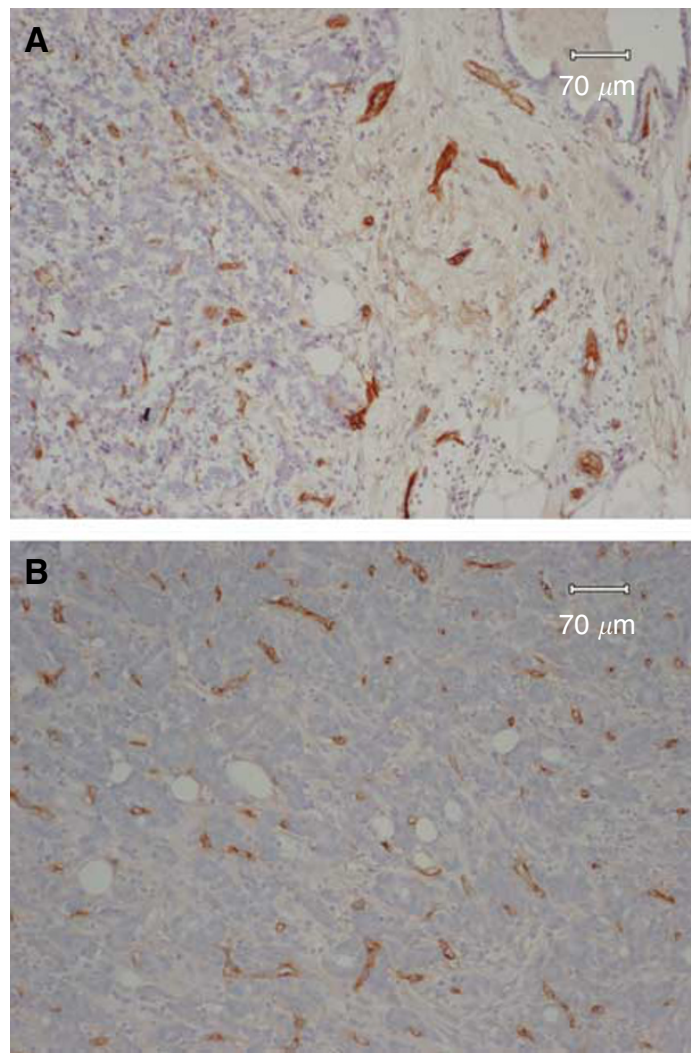

Figure I Microvessel staining: microvessels were highlighted by staining endothelial cells (staining for factor VIII-related antigen). (A) Example of an area from a tumour with AMC. Microvessels were highlighted by staining endothelial cells along the border between cancer nests and stroma. (B) Representative field of HMC showing high vascularisation.

Microvessels were counted by one investigator (TK). To evaluate the most effective method to quantify MVD in angiogenesis, two different methods were tried. The first was average microvessel count (AMC) per square millimetre (Kato et al, 1999, 2003). One maximal area of all the cut surfaces exhibiting invasive components in each tumour was scanned at high power $(200 \times)$ and the number of microvessels in all the areas along the border between cancer nests and the stroma was recorded (Figure 1A). The average number of microvessels in all the fields scanned at high power was calculated giving the mean AMC. The other method was to use the three highest microvessel counts (HMC) per square millimetre (Figure 1B). These criteria were similar to those of Weidner et al (1991). The patients were divided into two groups (low or high) according to the median AMC or HMC value $\left(54.4,85.3 \mathrm{~mm}^{-2}\right.$, respectively) of all patients.

\section{Statistical analysis}

Statistical analysis of the data was performed with the Survival Tools for Statview-J 5.0 package (Abacus Concepts, Berkeley, CA, USA). All tests were two-tailed. For comparison of the median follow-up duration and the two groups and for association with T-stage, surgical treatment, ER status, lymph-node status, grade, vascular invasion and histologic type and the two groups, Mann-Whitney U-test, $\chi^{2}$-test or Fisher's exact tests was used. For comparison of mean age, tumour size and MVD and the two groups or four groups Mann-Whitney U-test or Kruskal-Wallis test was used. We examined the univariate relationships between prognostic indicators and relapse-free survival (RFS) and overall survival (OS) by fitting Kaplan - Meier survival curves (Kaplan and 
Meier, 1958) to various levels of the prognostic indicators and then looked for differences among the curves using the log-rank test (Mantel, 1966). The Cox proportional hazards regression model was also used for the multivariate analysis (Cox, 1972).

\section{RESULTS}

\section{Characteristics and survival of the 436 original patient} population

Two hundred and seventeen Japanese and 219 British patients underwent breast cancer surgery in Tokyo and Oxford. In the original series, British patients were significantly older $(56.5 \pm 11.3$ years vs $52.4 \pm 12.6$ years, $P<0.01$ ) and had smaller tumours $(2.1 \pm 1.6$ vs $2.6 \pm 2.9 \mathrm{~cm} ; P<0.01)$ and had more ER-positive tumours $(77.2$ vs $56.3 \%, P<0.01)$ compared with Japanese patients, but there was no difference in the prevalence of lymph node metastasis (37.9 vs 36.4\%, $P=0.74)$. Cox regression modelling was used to determine the effect of population and other variables on RFS and OS. British patients had a significantly worse RFS and OS compared with Japanese patients $(P=0.01$, $P=0.01$, respectively). The adjusted hazard ratio (HR) and $95 \%$ confidence intervals (CI) for RFS or OS for British patients compared with Japanese patients was $2.3(1.4-3.5)$ or $2.8(1.6-4.9)$ $(P<0.01, P<0.01$, respectively).

\section{Characteristics of the 357 patient subset for pathological analyses}

Because of lack of tumour tissue, only a subset could be analysed for MVD. The median follow-up duration of the Japanese and British patients was 76 months (range, 1-105) and 90 months

Table I Clinicopathological characteristics of Japanese and British patients

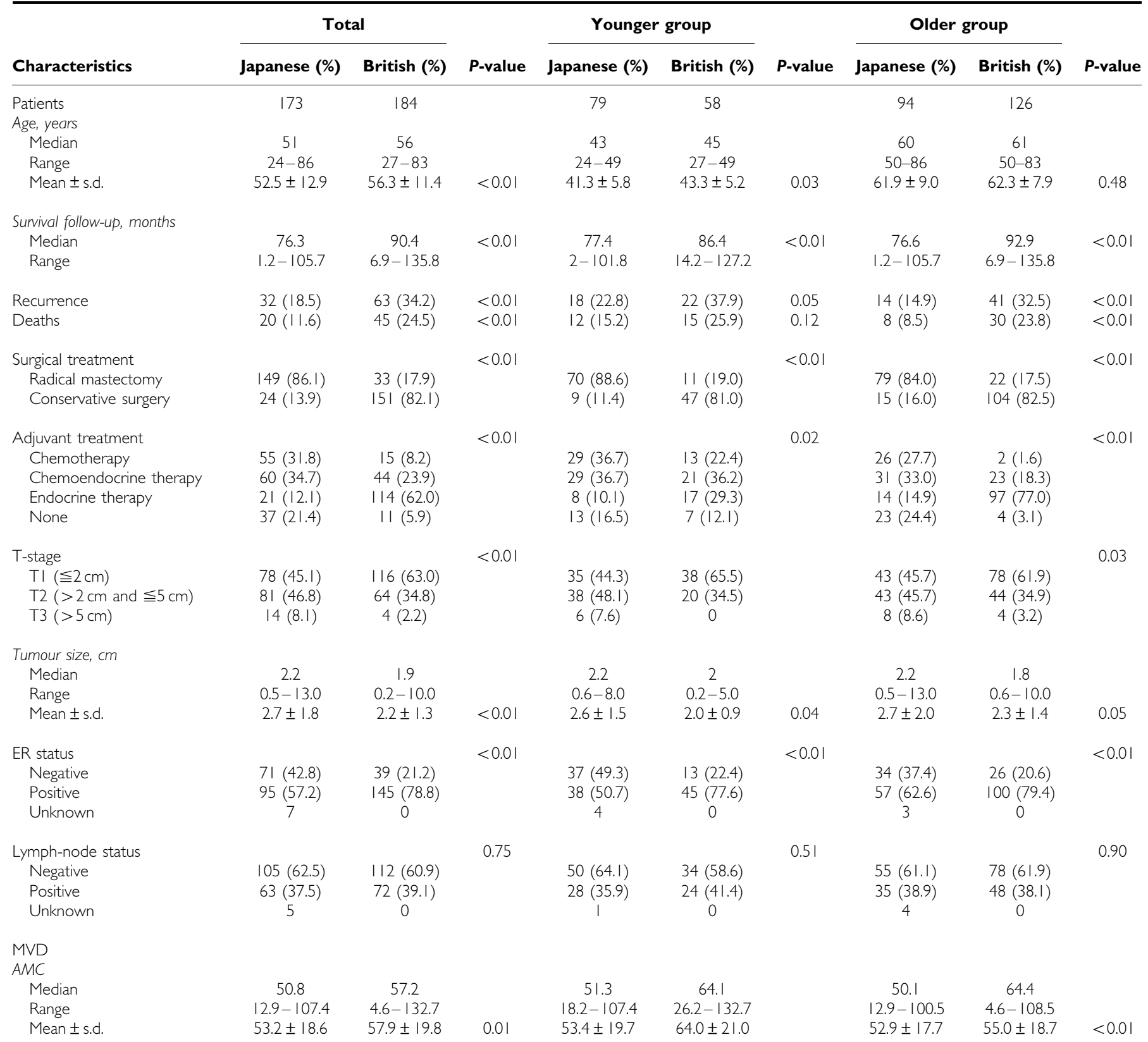


Table I (Continued)

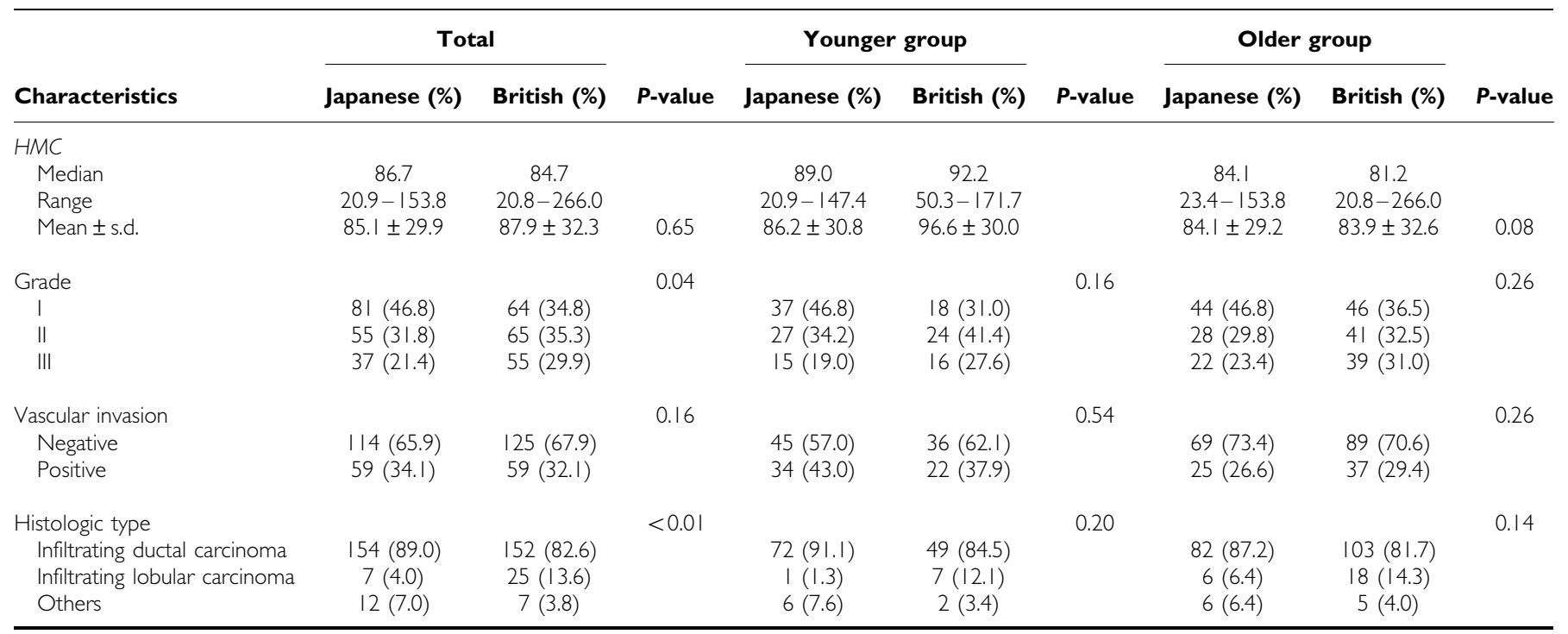

Younger group: age $<50$ years; Older group: age $\geqq 50$ years. $E R=$ oestrogen receptor; $M V D=$ microvessel density; $A M C=$ average microvessel counts; $H M C=$ highest microvessel counts. Younger group: age $<50$ years; Older group: age $\geqq 50$ years. Per cent do not include cases of unknown ER or lymph-node status.

(range, 7-135) $(P<0.01$; Table 1). Clinical and pathological data are listed in Table 1. Seven patients were lost to follow-up and one patient died within 1 year. The other cases were followed up for over 1 year. Japanese patients tended to be younger, with the peak in age distribution between 40 and 49 years of age, compared to 50-59 in British patients. Mean ages were also four to five years younger in Japanese women (means 56 and $52, P<0.01$; Table 1 ). Japanese patients had proportionately more T2 and T3 cases than did the British patients $(46.8,8.1$ vs $34.8,2.2 \%$, respectively, $P<0.01$, Table 1). The ratio of ER-positive British patients was significantly higher than that of Japanese patients $(78.8$ vs $57.2 \%$, $P<0.01$, Table 1). No difference in the prevalence of lymph-node metastasis was found between Japanese and British patients (39.1 vs $37.5 \%, P=0.75$; Table 1). British patients had a higher AMC compared with Japanese patients $(57.9 \pm 19.8$ vs 53.2 \pm 18.6 ; $P=0.01)$, but not HMC $(87.9 \pm 32.3$ vs $85.1 \pm 29.9, P=0.65$, Table 1). Moreover, younger British patients (age $<50$ years) had the highest AMC compared to both younger and older Japanese and older British patients $(P<0.01$, Table 1). Patients with high AMC had proportionately more infiltrating lobular carcinoma than did the patients with low AMC $(P=0.04)$. However, there was no correlation between high AMC and other variables such as T-stage, ER-positivity, age, lymph-node metastasis, high grade or vascular invasion. Japanese patients had proportionately more grade I cases than did the British patients ( 46.8 vs $34.8 \%, P=0.04$, Table 1$)$, however, there was no correlation between vascular invasion and the population $(P=0.16)$. There were significant differences in the surgical treatment - for example $86.1 \%$ of Japanese patients had radical or modified radical mastectomy vs $17.9 \%$ of British patients $(P<0.01)$, and conversely $13.9 \%$ of Japanese patients had conservative surgery (local excision alone or local excision and radiotherapy) $v s 82.1 \%$ of British patients $(P<0.01$; Table 1$)$. $6.5 \%$ of Japanese patients received adjuvant chemo- or chemoendocrine therapy vs $32.1 \%$ of British patients $(P<0.01)$, and $12.1 \%$ of Japanese patients had adjuvant hormone therapy alone $v s 62.0 \%$ of British patients $(P<0.01)$. Japanese patients were proportionately more likely to receive chemotherapy than endocrine therapy.
Relationship between prognostic variables and relapse-free survival (RFS) and overall survival (OS)

British patients had a significantly worse RFS and OS compared with Japanese patients ( $P=0.01, P=0.02$, respectively; Figures $2 \mathrm{~A}$ and $\mathrm{B}$ ). For patients in T2 stage subgroup, British patients had a significantly worse RFS and OS than Japanese patients $(P<0.01$, $P<0.01$, respectively; Figures $3 \mathrm{~A}$ and $\mathrm{B})$. Also for patients in the T3 stage subgroup, British patients had a significantly worse RFS compared with Japanese patients $(P=0.04)$, but not a worse OS $(P=0.12$; curves not shown). But for patients in T1 subgroup there was no difference $(P=0.19, P=0.17$, respectively; curves not shown). For breast cancer stratified by ER status, ER-negative British patients had a worse OS than Japanese patients $(P=0.04$; curves not shown), but not a worse RFS $(P=0.10)$. In contrast, there was no significant difference in RFS and OS amongst Japanese and British women with ER-positive cases $(P=0.14$, $P=0.27$, respectively; curves not shown). For patients in the older subgroup, British patients had a significantly worse RFS and OS than Japanese patients $(P=0.03, P=0.02$, respectively; Figures $4 \mathrm{~A}$ and $\mathrm{B})$ but not for patients in younger subgroup $(P=0.15$, $P=0.50$, respectively; curves not shown). Moreover, RFS and OS for ER-negative British older patients was worse than for Japanese $(P=0.04, P=0.01$, respectively; Figures $4 \mathrm{C}$ and $\mathrm{D})$, while there was no significant difference in RFS and OS amongst ER-positive Japanese and British older women $(P=0.24, P=0.29$, respectively; curves not shown).

\section{Univariate and multivariate analyses of relapse-free survival (RFS) and overall survival (OS)}

Model 1 of Table 2 indicates that British patients had a significantly worse RFS and OS compared with Japanese patients (HR: 2.1, 2.4, $P<0.01, P<0.01$, respectively) and patients with T2 or T3-stage and grade III had a significantly worse RFS and OS compared with the patients with T1-stage and grade I. However, AMC was not associated with RFS and OS in either analyses. British patients also had a significantly worse RFS and OS compared with Japanese patients in the model 2 (HR: 2.6, 3.2; 

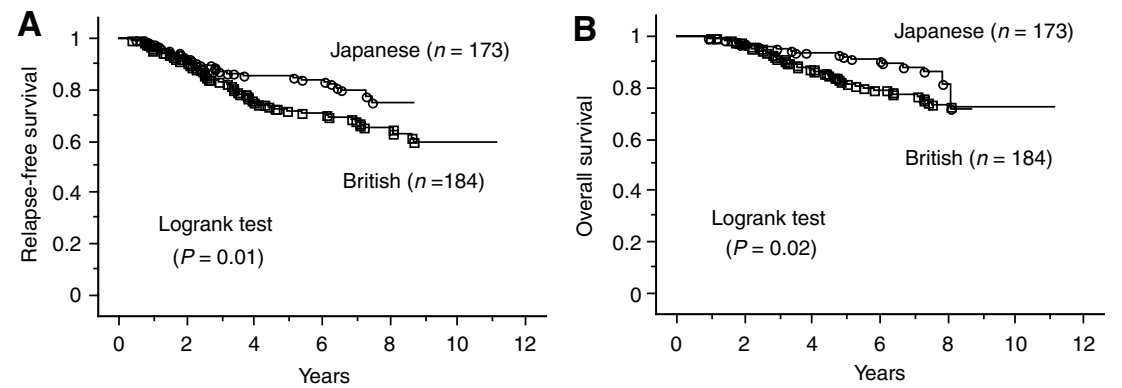

Figure 2 Kaplan-Meier survival curves for all patients with breast cancer.

(A) Relapse-free survival stratified by population.

Overall survival related to population.
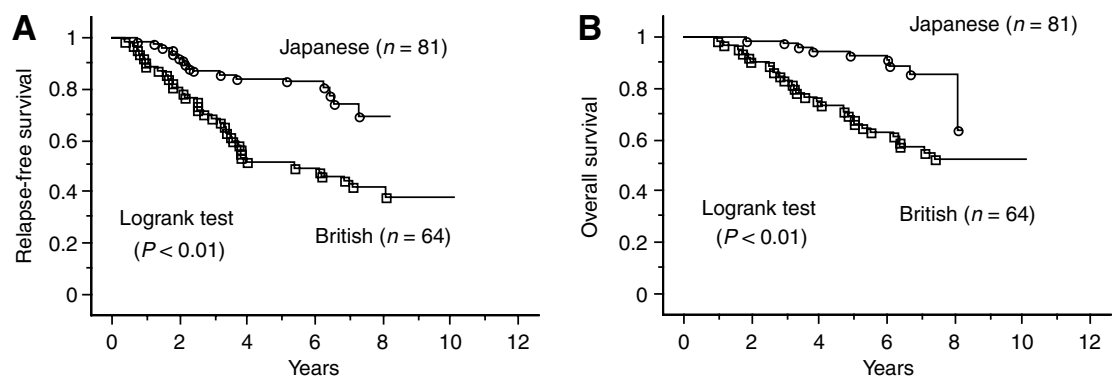

Figure 3 Kaplan-Meier survival curves for all patients with T2 tumours. (A) Relapse-free survival for the patients with T2 tumours stratified by population. (B) Overall survival for the patients with $\mathrm{T} 2$ tumours related to population.
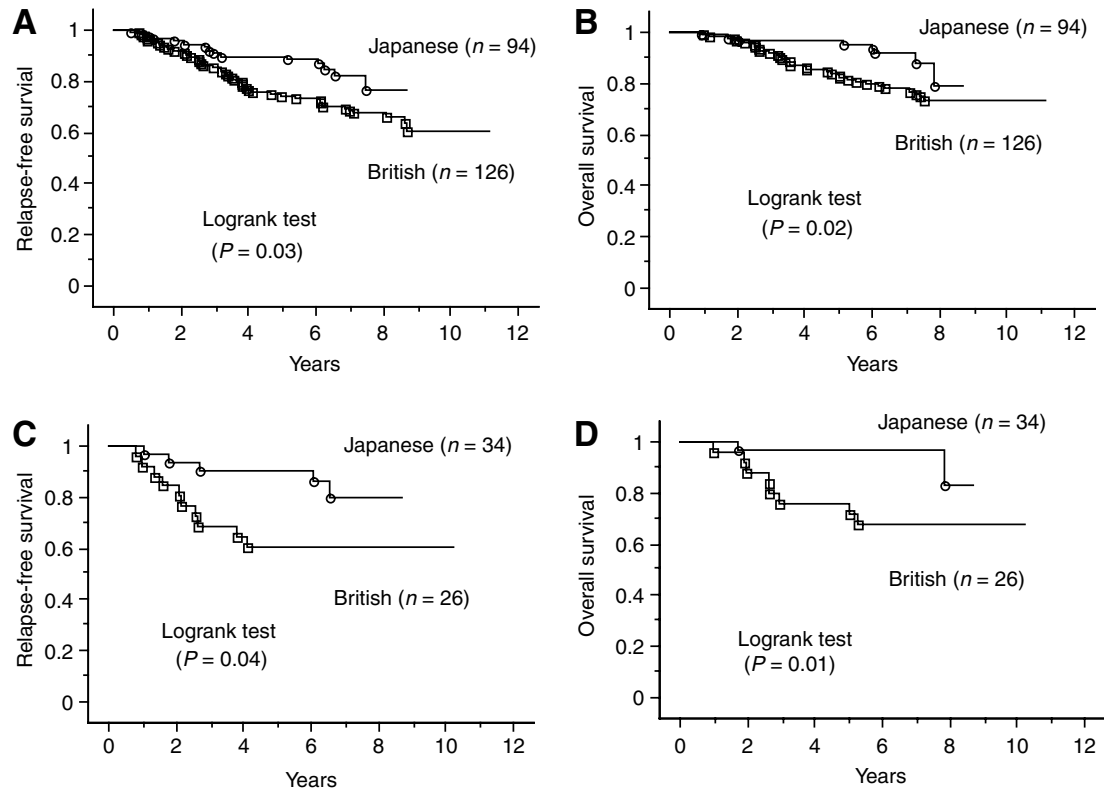

Figure 4 Kaplan-Meier survival curves for older patients with breast cancer. (A) Relapse-free survival I for all older patients stratified by population. (B) Overall survival for all older patients related to population. (C) Relapse-free survival I for ER-negative older patients stratified by population. (D) Overall survival for ER-negative older patients related to population.

$P<0.01, P<0.01$, respectively). In particular, British patients with T2 tumours had significantly worse RFS and OS compared with Japanese women (HR: $3.6,5.0 ; P<0.01, P<0.01$, respectively; Table 3). In the T-2 stage subgroup, lymph-node status was significantly associated with RFS and OS (HR: 2.4, 2.1; $P<0.01$, $P=0.03$, respectively; Table 3 ). Table 4 shows the unadjusted hazard ratio for age and population with multivariate adjusted hazard ratio being controlled for T-stage, nodes status, ER status, grade, AMC, and vascular invasion. The unadjusted hazard ratio for RFS or OS for older British patients compared with older Japanese patients was 1.9 or $2.4(P=0.03, P=0.03$, respectively, Table 4). After controlling for other variables, the hazard ratio of RFS or OS for them was 3.2 or $3.9(P<0.01, P<0.01$, respectively, Table 4). Older Japanese patients had better outcomes than older 
Table 2 Multivariate analysis of the value of prognostic factors for relapse-free survival and overall survival among all Japanese and British patients

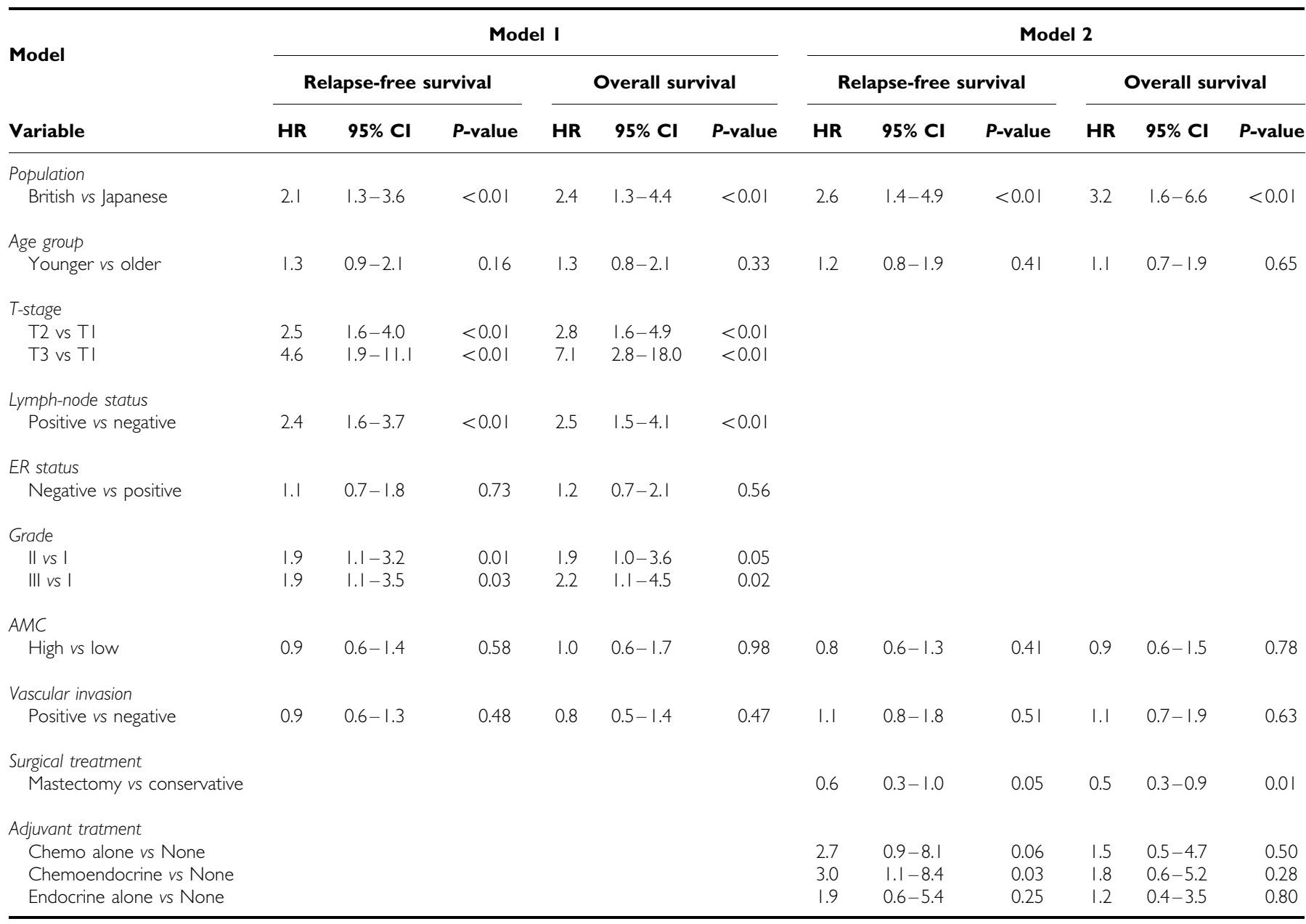

$A M C=$ average microvessel counts; $E R=$ estrogen receptor; $\mathrm{HR}=$ hazards ratio; $95 \% \mathrm{Cl}=95 \%$ confidence interval. Younger group: age $<50$ years; Older group: age $\geqq 50$ years Multivariate model I adjusted for population, age, T-stage, lymph-node status, ER status, grade, AMC and vascular invasion. Multivariate model 2 adjusted for population, age, AMC, vascular invasion, surgical treatment and adjuvant treatment. Hazards ratio from Cox regression analysis.

British patients, while there were no significant differences among younger Japanese and British patients. In the low AMC subgroup, the population and T-stag were significantly associated with RFS and OS in multivariate analysis (model 1 of Table 5). In contrast, in the high AMC subgroup, although T-stage, lymph-node status and grade significantly affected the association with RFS and OS, population had no effect on RFS and OS (model 2 of Table 5).

\section{DISCUSSION}

Several investigators have found that Japanese patients with breast cancer have a better survival than American or British patients (Wynder et al, 1963; Morrison et al, 1976; Friedel et al, 1991). There are several limitations to these hospital-based studies. There is often insufficient uniformity amongst populations. There might have been a selection bias. In the previous studies, none used multivariate methods to simultaneously control the other prognostic variables. Thus, it is not known whether these survival differences were due to race or tumour variables. Our results, however, do show the better outcome in older Japanese compared with older British patients before and after adjusting for other variables (Table 4). Only other study showed that the difference in survival was restricted to postmenopausal patients (Sakamoto et al, 1981). Age-adjusted mortality rates in England and Wales started to decrease in the late 1980s (Beral et al, 1995), while those in Japan have gradually increased, but still lower than the former rates (Tominaga and Kuroishi, 1995; The Research Group for Population-based Cancer Registration in Japan, 2003) As there has been a gradual improvement in survival in British patients, the magnitude of survival differences reported in this study was not nearly as great as that reported in a previous study (Merchant et al, 1999). Improved survival from this cancer in the UK may be due to increased social interest, advances in diagnosis according to the NHS Breast Screening Programme, and development of adjuvant endocrine therapy and chemotherapy (Beral et al, 1995; Kobayashi, 2004).

There is also strong evidence to suggest a population-dependent variation in the biology of breast cancer, such as ER status, p53 gene mutation, HER-2/neu, S-phase fraction, lymphatic infiltration, sinus histiocytosis, and certain histological patterns (Sakamoto et al, 1981; Friedel et al, 1991; Elledge et al, 1994; Merchant et al, 1999). Differences in these biologic characteristics do not explain the relatively high survival rate of Japanese breast cancer patients. According to the study of Braun et al (2004), native Hawaiian patients had a poor survival in spite of a high ER positivity rate. The current study also showed that British patients had a high percentage of ER-positive tumours and an increased risk of death, in spite of the extensive use of adjuvant endocrine therapy. In our study the pathological variables do not explain why the survival of 
British patients is poorer than Japanese patients, as the former had smaller and more ER-positive tumours than the latter and the prevalence of lymph-node involvement was also the same. Therefore, we investigated MVD as a possible mechanism, because the Japanese diet is rich in antioxidants and green tea, consumed many times daily by the average Japanese and contains active antiangiogenic substances (Sartippour et al, 2002; Rodriguez et al, 2006). Thus, dietary differences involving these agents was a possible factor.

Table 3 Multivariate analysis of the value of prognostic factors for relapse-free survival and overall survival among Japanese and British patients with $\mathrm{T} 2$ tumours

\begin{tabular}{|c|c|c|c|c|c|c|}
\hline \multirow[b]{2}{*}{ Variable } & \multicolumn{3}{|c|}{ Relapse-free survival } & \multicolumn{3}{|c|}{ Overall survival } \\
\hline & HR & $95 \% \mathrm{Cl}$ & $P$-value & HR & $95 \% \mathrm{Cl}$ & $P$-value \\
\hline \multicolumn{7}{|l|}{ Population } \\
\hline British vs Japanese & 3.6 & $1.9-6.9$ & $<0.01$ & 5.0 & $2.1-11.8$ & $<0.01$ \\
\hline \multicolumn{7}{|l|}{ Age group } \\
\hline Younger vs older & 1.1 & $0.6-1.9$ & 0.82 & 1.1 & $0.5-2.2$ & 0.81 \\
\hline \multicolumn{7}{|l|}{ Lymph-node status } \\
\hline Positive vs negative & 2.4 & $1.4-4.3$ & $<0.01$ & 2.1 & $1.1-4.1$ & 0.03 \\
\hline \multicolumn{7}{|l|}{ ER status } \\
\hline Negative vs positive & 1.6 & $0.8-3.1$ & 0.20 & 2.0 & $0.9-4.6$ & 0.08 \\
\hline \multicolumn{7}{|l|}{ Grade } \\
\hline || vs I & 1.7 & $0.6-3.5$ & 0.14 & 1.6 & $0.6-4.0$ & 0.30 \\
\hline ||l vs I & 1.2 & $0.5-2.8$ & 0.65 & 1.5 & $0.6-4.1$ & 0.42 \\
\hline \multicolumn{7}{|l|}{$A M C$} \\
\hline High vs low & 0.7 & $0.4-1.3$ & 0.24 & 0.8 & $0.4-1.6$ & 0.45 \\
\hline \multicolumn{7}{|l|}{ Vascular invasion } \\
\hline Positive vs negative & 1.1 & $0.6-1.9$ & 0.78 & 1.0 & $0.5-2.0$ & 0.98 \\
\hline
\end{tabular}

To our knowledge, this is the first study to compare the prognostic value of microvessel density in Japanese and British patients with invasive breast cancer. Various techniques for the evaluation of neovascularisation have been tested in breast cancer. Some studies have suggested that microvessel density was representative of angiogenesis of the tumour and was an independent and highly significant prognostic factor (Weidner et al, 1991; Horak et al, 1992; Gasparini et al, 1994; Kato et al, 2001; Tsutsui et al, 2003). However, other authors reported that angiogenesis did not predict recurrence in patients with primary breast cancer (van Hoef et al, 1993; Goulding et al, 1995). The significance of angiogenesis remains controversial due to studies which varied in patient selection, length of time of patient followup, antibody used to detect endothelial cells, sample size, method of counting microvessels, and the race. Some clinical studies suggested that to use an antibody to CD31 may be superior to using factor VIII-related antigen (Horak et al, 1992; Fox et al, 1995); however, another study reported that this greater sensitivity of anti-CD31 of vascular endothelium did not yield results more discriminating for predicting survival outcome than results produced with factor VIII-related antigen (Gasparini et al, 1994). Using an antibody to the latter antigen, many stromal vessels can be stained well as we published previously (Kato et al, 1999). When we compared AMC with the highest microvessel density in one or three fields (the hot spots) in the previous study, AMC was a more reliable factor than that of the hot spots (Kato et al, 1999). The results show that AMC was an independent prognostic factor, but its prognostic impact was not as strong as lymph-node status and clinical tumour size. The method of Weidner et al (1991) is popular, however, we have questioned whether or not the hot spots in one slide per tumour is representative for the whole tumour. Selection of the hot-spots area for counting is one of the possible sources of this discordance and it requires the judgment of the investigators to select which region is the most vascular (Kato et al, 1999, 2003; Medri et al, 2000). Both the Chalkley method and computerised imaging analysis may reduce the subjectivity of evaluation in quantification of microvessel count (Fox et al, 1995; Gasparini, 2001; Hansen et al, 2004). In a prior study, Kato et al (1999) showed that the area of highest microvessels most frequently $(76.5 \%)$ appeared at the margins of the carcinoma, where invasion is taking place and active growth most likely and they counted the number of the microvessels along

Table 4 Age-specific models among British patients compared with Japanese patients

\begin{tabular}{|c|c|c|c|c|c|c|}
\hline Variable & \multicolumn{3}{|c|}{ Relapse-free survival } & \multicolumn{3}{|c|}{ Overall survival } \\
\hline \multicolumn{7}{|l|}{ Older group (Age $\geqq 50$ years) } \\
\hline Population (British vs Japanese) & 1.9 & $1.1-3.6$ & 0.03 & 24 & $1.1-5.2$ & \\
\hline AMC (high vs low) & 1.2 & $0.7-2.1$ & 0.41 & 1.4 & $0.7-2.7$ & 0.27 \\
\hline \multicolumn{7}{|l|}{ Multivariate adjusted } \\
\hline AMC (high vs low) & 1.3 & $0.7-2.3$ & 0.35 & 1.3 & $0.7-2.5$ & 0.46 \\
\hline \multicolumn{7}{|l|}{ Younger group (Age $<50$ years) } \\
\hline \multicolumn{7}{|l|}{ Unadjusted } \\
\hline Population (British vs Japanese) & 1.6 & $0.8-2.9$ & 0.15 & 1.4 & $0.6-3.1$ & 0.35 \\
\hline AMC (high vs low) & 0.6 & $0.3-1.1$ & 0.12 & 0.8 & $0.4-1.7$ & 0.53 \\
\hline \multicolumn{7}{|l|}{ Multivariate adjusted } \\
\hline
\end{tabular}

$\mathrm{AMC}=$ average microvessel counts; $\mathrm{ER}=$ oestrogen receptor; $\mathrm{HR}=$ hazards ratio; $95 \% \mathrm{Cl}=95 \%$ confidence interval. Multivariate models adjusted for population, T-stage, lymph-node status, ER status, grade, AMC and vascular invasion. Hazards ratio from Cox regression analysis. 
Table 5 Multivariate analysis of the value of prognostic factors for relapse-free survival and overall survival among Japanese and British patients with low and high AMC tumours

\begin{tabular}{|c|c|c|c|c|c|c|c|c|c|c|c|c|}
\hline \multirow{2}{*}{$\begin{array}{l}\text { Model } \\
\text { Variable }\end{array}$} & \multicolumn{6}{|c|}{ Model I (low AMC) } & \multicolumn{6}{|c|}{ Model 2 (high AMC) } \\
\hline & \multicolumn{3}{|c|}{ Relapse-free survival } & \multicolumn{3}{|c|}{ Overall survival } & \multicolumn{3}{|c|}{ Relapse-free survival } & \multicolumn{3}{|c|}{ Overall survival } \\
\hline \multicolumn{13}{|l|}{ Population } \\
\hline British vs Japanese & 3.1 & $1.5-6.4$ & $<0.01$ & 3.3 & $1.3-8.2$ & $<0.01$ & 1.7 & $0.8-3.4$ & 0.14 & 1.7 & $0.8-3.9$ & 0.19 \\
\hline \multicolumn{13}{|l|}{ T-stage } \\
\hline T2 vs TI & 3.0 & $1.5-5.8$ & $<0.01$ & 3.9 & $1.7-8.8$ & $<0.01$ & 2.5 & $1.3-4.8$ & $<0.01$ & 2.5 & I. I-5.4 & 0.02 \\
\hline T3 vs TI & 1.7 & $0.3-8.2$ & 0.49 & 3.9 & $0.7-20.2$ & 0.10 & 22.1 & $6.4-76.4$ & $<0.01$ & 15.3 & $4.3-54.7$ & $<0.01$ \\
\hline \multicolumn{13}{|l|}{ Lymph-node status } \\
\hline Positive vs negative & 2.5 & 1.4-4.4 & $<0.01$ & 1.9 & $0.9-4.0$ & 0.06 & 2.1 & $1.1-3.9$ & 0.02 & 2.9 & $1.3-6.3$ & $<0.01$ \\
\hline || vs I & 1.5 & $0.7-3.1$ & 0.25 & 1.4 & $0.6-3.3$ & 0.48 & 2.8 & $1.3-6.2$ & 0.01 & 3.1 & $1.2-8.4$ & 0.02 \\
\hline |II vs I & 1.5 & $0.6-3.4$ & 0.36 & 1.5 & $0.6-4.0$ & 0.38 & 3.9 & $1.6-9.4$ & $<0.01$ & 4.2 & $1.4-12.9$ & 0.01 \\
\hline \multicolumn{13}{|l|}{ Vascular invasion } \\
\hline Positive vs negative & 1.1 & $0.6-1.9$ & 0.78 & 0.9 & $0.4-1.9$ & 0.73 & 0.6 & $0.3-1.2$ & 0.12 & 0.8 & $0.3-1.7$ & 0.49 \\
\hline
\end{tabular}

the border between cancer nests and the stroma (Figure 1A). The average number of microvessels in all the fields scanned at high power was calculated giving the mean AMC. We think that the method of determining AMC might be a more objective method than that of the hot spots because if the edge of the tumour for counting is decided, it is not so difficult to count the vessels, although it was somewhat subjective to determine the edge of the tumour with either ductal carcinoma in situ or lobular carcinoma in situ for counting. Thus, the significance of the methods for evaluation of microvessels remains controversial.

In the current study, to investigate the associations of microvessel density and other variables with survival, and whether they correlate with survival differences between Japanese and British patients, we used the methods of both Weidner et al (1991) and Kato et al (1999) between Japanese and British patients. British patients had a higher AMC compared with Japanese patients, but not HMC. In particular, younger British patients had the highest AMC of the 4 groups. AMC appears to be a more useful surrogate marker of tumour angiogenesis than HMC in this series. AMC and HMC were used as continuous variables at first to investigate the associations of microvessel density and other variables with survival. They were also divided into either two or three categories to analyse, but this did not significantly change the lack of association with survival. Population was significantly associated with RFS and OS in the low AMC subgroup, but had no effect on RFS and OS in high AMC subgroup. T-stage, lymph-node status and grade significantly affected the association with RFS and OS in the latter subgroup. Thus, there was no Japanese-British disparity in the more aggressive breast tumours with high AMC, tumours more than $2 \mathrm{~cm}$, positive nodes or high grade. The present study shows that AMC contributes to the Japanese-British disparity in breast cancer. However, this study does not explain the favourable prognosis for Japanese patients, especially, for those who are older.

The administration of systemic adjuvant chemotherapy or endocrine therapy can improve RFS and OS in breast cancer patients (Early Breast Cancer Trialist' Collaborative Group, 1992). ER status is used to inform breast cancer treatment, and patients with ER-positive tumours are candidates for tamoxifen or similar agents. In contrast, patients with ER-negative tumours are often given chemotherapy. Boyer-Chammard et al (1999) reported that African-American, Hispanic, and Asian patients with breast cancer from 1984 through 1990 were more likely than Caucasian patients to receive chemotherapy and less likely to receive endocrine therapy. The current study is similar to those results. The study by the Collaborative Study Group of Adjuvant Chemoendocrine Therapy for Breast Cancer (ACETBC) in Japan was carried out from 1982 (Kasumi et al, 2003; Noguchi et al, 2005). Most of the Japanese patients in this study were treated according to that regimen. As Japanese patients had more ER-negative tumours than British patients, they were more likely to receive chemotherapy than endocrine therapy. Treatment may be a contributing factor on survival between populations. There were clear differences in the treatment policies. After controlling for population, age, AMC and vascular invasion, surgical treatment affected the association with the survival, but adjuvant treatment had no effect on the survival (Table 2). However, patients who received chemotherapy alone had significantly better prognosis than those who had no adjuvant therapy in ER-negative subgroup alone (RR: 0.1, 95\% CI: $0.02-0.8, P=0.03$ ).

There are several limitations to the current study. Firstly, it is not certain that the cases are comparably representative of the population cases in each country and there might have been a selection bias. However the patients came from the population local to their main cancer centres. Secondly, medical care procedures and patterns differ significantly in each country. Thirdly, there is the possibility that any systemic differences in the handling of tissue may well affect the immunohistochemical results. Fourthly, as the number of the cases we studied is less than the original selected Japanese and British cases, there may be some bias, but distribution of age, tumour size, ER status and survival in the original cases was similar to those of the cases in the pathology 
analysis. Finally, the length of time of follow-up varied according to the two groups, but this would not affect the survival curves for first 5 years. The advantage of this study includes consistency in the antibody used to detect endothelial cells and the methods of counting microvessels, that is same polyclonal antibody for factor VIII-related antigen was used. All slides of both Japanese and British patients for $\mathrm{H} \& \mathrm{E}$ and factor VIII-related antigen staining were performed according to the same method and at the same institute (John Radcliffe Hospital, Headington, Oxford, UK).

Overall therefore this study shows the difference between populations occurs in the older age group, not younger, and in the population with low AMC. Although younger British patients had the highest AMC, their prognosis was not worse than the Japanese younger patients. In the smallest tumours again there was no population difference in survival, only in the T2 stage tumours was this seen. For those with ER-positive tumours there was no difference in relapse-free or overall survival and numbers are small with this subgroup analysis. However it appears that ER-negative British patients had much worse survival than ER-negative

\section{REFERENCES}

Axelsson K, Ljung BME, Moore II DH, Thor AD, Chew KL, Edgerton SM, Smith HS, Mayall BH (1995) Tumour angiogenesis as a prognostic assay for invasive ductal breast carcinoma. J Natl Cancer Inst 87: $997-1008$

Beral V, Hermon C, Reeves G, Peto R (1995) Sudden fall in breast cancer death rates in England and Wales. Lancet 345: 1642-1643

Boyer-Chammard A, Tayler TH, Anton-Culver H (1999) Survival differences in breast cancer among racial/ethnic groups: a population-based study. Cancer Detect Prev 23: 463-473

Braun KL, Fong M, Gotay C, Pagano IS, Chong C (2004) Ethnic difference in breast cancer in Hawaii: age, stage, hormone receptor status, and survival. Pacific Health Dialog 11: 146-153

Chaudary MA, Hayward JL, Bulbrook RD, Yoshida M, Miura S, Murai JT, Takatani O (1991) A comparison of epidemiological characteristics in breast cancer patients and normal patients in Great Britain and Japan: results of a prospective study. Breast Cancer Res Treat 18: S19-S22

Claus EB, Rish NJ, Thompson WD (1990) Age at onset as an indicator of familiar risk of breast cancer. Am J Epidemiol 131: 961-972

Costello P, McCann A, Carney DN, Dervan PA (1995) Prognostic significance of microvessel density in lymph node negative breast carcinoma. Hum Pathol 26: $1181-1184$

Cox DR (1972) Regression models and life tables. J R Stat Soc B 34: 187-202

Early Breast Cancer Trialist' Collaborative Group (1992) Systemic treatment of early breast cancer by hormonal, cytotoxic, or immune therapy. 133 randomised trials involving 31000 recurrences and 24000 deaths among 75000 patients. Lancet 339: 1-15

Elledge RM, Clark GM, Chamness GC, Osborne CK (1994) Tumor biologic factors and breast cancer prognosis among white, Hispanic, and black patients in the United States. J Natl Cancer Inst 86: 705-712

Elston CW, Ellis IO (1991) Pathological prognostic factors in breast cancer. I. The value of histological grade in breast cancer: experience from a large study with long-term follow-up. Histopathol 19: 403-410

Fox S, Leek RD, Weekes MP, Whitehouse RM, Gatter KC, Harris AL (1995) Quantitation and prognostic value of breast cancer angiogenesis: comparison of microvessel density, Chalkley count, and computer image analysis. J Pathol 177: 275-283

Friedel GH, Millis RR, Sato T, Suichi T, Shikata I, Chaudary MA, Hayward JL (1991) Breast cancer in English and Japanese patients: prognostic significance of sinus histiocytosis and germinal center hyperplasia in axillary lymph nodes. Breast Cancer Res Treat 18: S73-S76

Gasparini G, Weidner N, Bevilacqua P, Maluta S, Palma PD, Caffo O, Barbareschi M, Boracchi P, Marubini E, Pozza F (1994) Tumour microvessel density, p53 expression, tumour size, and peritumoural lymphatic vessel invasion are relevant prognostic markers in nodenegative breast carcinoma. J Clin Oncol 12: 454-466

Gasparini G (2001) Clinical significance of determination of surrogate markers of angiogenesis in breast cancer. Oncol Hematol 37: 97-114
Japanese patients. The relation to ER suggest that the growthstimulating pathways for ER-negative cases may be different in the two populations perhaps modified by diet. Also since the difference applied to ER-negative patients, the much greater use of chemotherapy may have helped improve outcome. It will be interesting to compare a more recent series from within the last 5 years, to see if this difference has been reduced due to much greater use of chemotherapy currently in this group, ER negative over 50 s, in the UK.

These findings suggest that a haematogenous pattern of dissemination may be more important than a lymphatic one for international disparities in breast cancer outcomes. It can be speculated that the other surrogate markers of tumour angiogenesis or other biological variables for haematogenous dissemination or the growth pathways in relation to ER-negative status contribute to the worse survival of British patients. Further research into the biological behaviour of international differences and their effect on the survival of patients with breast cancer should be continued.
Gordon NH, Crowe JP, Brumberg DJ, Berger NA (1992) Socioeconomic factors and race in breast cancer recurrence and survival. Am J Epidemiol 135: $609-618$

Goulding H, Rashid NFNA, Robertson JF, Bell JA, Elston CW, Blamey RW, Ellis IO (1995) Assessment of angiogenesis in breast carcinoma: An important factor in prognosis? Hum Pathol 26: 1196-1200

Hansen S, Sorensen FB, Vach W, Grabau DA, Back M, Rose C (2004) Microvessel density compared with Chalkley count in a prognostic study of angiogenesis in breast cancer patients. Histopathology 44: 428-436

Horak ER, Leek R, Klenk N, Lejeune S, Smith K, Stuart N, Greenall M, Stepniewska K, Harris AL (1992) Angiogenesis, assessed by platelet/ endothelial cell adhesion molecule antibodies, as indicator of node metastases and survival in breast cancer. Lancet 340: 1120-1124

Iscovich JM, Iscovich RB, Howe G, Shiboski S, Kaldor JM (1989) A case-control study of diet and breast cancer in Argentina. Int J Cancer 44: 770-776

Kaplan EL, Meier P (1958) Non-parametric estimation from incomplete observations. J Am Stat Assoc 53: 457-481

Kasumi F, Yoshimoto M, Uchino J, Abe R, Nomura Y, Sugimachi K, Nakazato H, Abe O (2003) Meta-analysis of five studies on Tegafur plus Uracil (UFT) as post-operative adjuvant chemotherapy for breast cancer. Oncology 64: 146-153

Kato T, Kameoka S, Kimura T, Nishikawa T, Kobayashi M (2003) The combination of angiogenesis and blood vessel invasion as a prognostic indicator in primary breast cancer. Br J Cancer 88: 1900-1908

Kato T, Kameoka S, Kimura T, Soga N, Abe Y, Nishikawa T, Kobayashi M (2001) Angiogenesis as a predictor of long-term survival for 377 Japanese patients with breast cancer. Breast Cancer Res Treat 70: 65-74

Kato T, Kimura T, Miyakawa R, Nobue I, Fujii A, Yamamoto K, Kameoka S, Toshio N, Kasajima T (1999) The methodology of quantitation of microvessel density and prognostic value of neovascularization associated with long-term survival in Japanese patients with breast cancer. Breast Cancer Res Treat 53: 19-31

Kawamura T, Sobue T (2005) Comparison of breast cancer mortality in five countries: France, Italy, Japan, the UK and the USA from the WHO mortality database (1960-2000). Jpn J Clin Oncol 35: 758-759

Kobayashi S (2004) What caused the decline in breast cancer mortality in the United Kingdom? Breast Cancer 11: 156-159

Mantel N (1966) Evaluation of survival data and two new rank order statistics arising in its consideration. Cancer Chemother Rep 50: $163-170$

Medri L, Nanni O, Volpi A, Scarpi E, Dubini A, Riccobon A, Becciolini A, Bianchi S, Amadori D (2000) Tumor microvessel density and prognosis in node-negative breast cancer. Int J Cancer 89: 74-80

Merchant WJ, Millis RR, Smith P, Chaudary MA, Barnes DM (1999) Expression of c-erbB2 and p53 protein is similar in breast cancer from British and Japanese patients. Int J Cancer 84: 278-283

Morrison AS, Lowe R, MacMahan B, Ravnihar B, Yuasa Shu (1976) Some international differences in treatment and survival in breast cancer. Int $J$ Cancer 18: $269-273$ 
Natarajan N, Nemoto D, Nemoto T, Mettlin C (1988) Breast cancer survival among Orientals and whites in the United States. J Surg Oncol 39: $206-209$

Noguchi S, Koyama H, Uchino J, Abe R, Miura S, Sugimachi K, Akazawa K, Abe O (2005) Postoperative adjuvant therapy with Tamoxifen, Tegafur plus Uracil, or both in patients with node-negative breast cancer: a pooled analysis of six randomized controlled trials. J Clin Oncol 23: $2172-2184$

Ogawa Y, Chung YS, Nakata B, Takatsuka S, Maeda K, Sawada T, Kato Y, Yoshikawa K, Sakurai M, Sowa M (1995) Microvessel quantitation in invasive breast cancer by staining for factor VIII-related antigen. $\mathrm{Br} J$ Cancer 71: $1297-1301$

Rodriguez SK, Guo W, Liu L, Band MA, Paulson EK, Meydani M (2006) Green tea catechin, epigallocatechin-3-gallate, inhibits vascular endothelial growth factor angiogenic signaling by disrupting the formation of a receptor complex. Int J Cancer 118: 1635-1644

Sakamoto G, Sugano H, Hartman W (1981) Comparative pathological study of breast carcinoma among American and Japanese. In: Breast Cancer, McGuire WL (ed) pp 211-231. Plenum Publishing Corporation: New York

Sartippour MR, Shao ZM, Heber D, Beatty P, Zhang L, Liu C, Ellis L, Liu W, Go VL, Brooks MN (2002) Green tea inhibits vascular endothelial growth factor (VEGF) induction in human breast cancer cells. J Nutr 132: $2307-2311$

Simon MS, Severson RK (1996) Racial differences in survival of female breast cancer in the Detroit metropolitan area. Cancer 77: 308-314
Tamakoshi K, Yatsuya H, Wakai K, Suzuki S, Nishio K, Lin Y, Niwa Y, Kondo T, Yamamoto A, Tokudome S, Toyoshima H, Tamakoshi A (2005) Impact of menstrual and reproductive factors on breast cancer risk in Japan: results of JACC study. Cancer Sci 96: 57-62

The research Group for Population-based Cancer Registration in Japan (1999) Cancer incidence Japan. Gann Monograph Cancer Res 47: 83-143

The research Group for Population-based Cancer Registration in Japan (2003) Cancer incidence and incidence rates in Japan in 1998: estimates based on data from 12 population based cancer registries. Jpn J Clin Oncol 33: $241-245$

Tominaga S, Kuroishi T (1995) Epidemiology of breast cancer. Breast Cancer 2: $1-7$

Tsutsui S, Kume M, Era S (2003) Prognostic value of microvessel density in invasive ductal carcinoma of the breast. Breast Cancer 10: $312-319$

Van Hoef MEHM, Knox WF, Dhesi SS, Howell A, Schor AM (1993) Assessment of tumour vascularity as a prognostic factor in lymph nodenegative invasive breast cancer. Eur J Cancer 29: $1141-1145$

Weidner N, Semple JP, Welch WR, Folkman J (1991) Tumour angiogenesis and metastasis-correlation in invasive breast carcinoma. $N$ Engl J Med 324: $1-8$

Wynder EL, Kajitani T, Kuno J, Lucas JC, DePalo A, Farrow J (1963) A comparison of survival rates between American and Japanese patients with breast cancer. Surg Gynecol Obstet 117: 196-200

Yonemoto RH (1980) Breast cancer in Japan and the United Statesepidemiology, hormone receptors, pathology, and survival. Arch Surg 115: $1056-1062$ 